Check for updates

Cite this: Mater. Chem. Front., 2019, 3, 1678

Received 2nd March 2019 Accepted 10th June 2019

DOI: 10.1039/c9qm00133f

rsc.li/frontiers-materials

\section{Electronic structures and elastic properties of a family of metal-free perovskites $\dagger$}

\author{
Kai Li, ${ }^{a}$ Li-Yuan Dong, ${ }^{b}$ Hao-Xiang Xu, ${ }^{b}$ Yan Qin, ${ }^{b}$ Zhi-Gang Li, ${ }^{a}$ \\ Muhammad Azeem, ${ }^{b}$ Wei Li iD *ab and Xian-He Bu iD *a
}

\begin{abstract}
The electronic structures and elastic properties of three isostructural, metal-free perovskite materials, $\left(\mathrm{C}_{4} \mathrm{~N}_{2} \mathrm{H}_{12}\right)\left(\mathrm{NH}_{4} \mathrm{X}_{3}\right) \cdot \mathrm{H}_{2} \mathrm{O}$ (PIP-X, $\left.\mathrm{X}=\mathrm{Br}, \mathrm{Cl}, \mathrm{l}\right)$, were examined using density functional theory (DFT) calculations and high-pressure synchrotron $\mathrm{X}$-ray diffraction experiments. The calculated band structures and density of states demonstrate that all the compounds possess large direct bandgaps of $5.34 \mathrm{eV}$ for PIP-Cl, $4.67 \mathrm{eV}$ for PIP-Br, and $4.13 \mathrm{eV}$ for PIP-I. With the bromide and iodide, the valence band maximum and conduction band minimum mainly arise from the 3p- and 3s-states of the halogens, whereas the conduction band minimum of the chloride is dominated by the s-states of the nitrogen from the ammonium. Such an inverse dependence of bandgaps on the halogen radius originates from the increased band dispersions because of reduced halogen electronegativity. In addition, the full elastic constants of these compounds were calculated using DFT which enables the systematic mapping of their Young's moduli, shear moduli and Poisson's ratios. The $\mathrm{N}-\mathrm{H} \ldots \mathrm{X}$ bond strength governed by the halogen radius is primarily responsible for the discrete modulus properties in these compounds. Notably, these metal-free perovskites constructed using hydrogen bonds exhibit comparable rigidity with their hybrid organic-inorganic counterparts assembled using coordination bonds. Furthermore, the highpressure synchrotron powder X-ray diffraction experiments were performed on PIP-Br, which not only validated the DFT results but also revealed its comparable stiffness to methylammonium lead bromine $\left(\mathrm{CH}_{3} \mathrm{NH}_{3} \mathrm{PbBr}_{3}\right)$ under hydrostatic stress.
\end{abstract}

\section{Introduction}

Perovskite materials are a broad class of $\mathrm{ABX}_{3}$-type compounds which have analogous structures to calcium titanate. ${ }^{1,2}$ They have been the subject of research for several decades because of their chemical diversity and multifunctionality which have enabled great achievements in optoelectronic applications. ${ }^{3-6}$ In terms of chemical composition, the evolution of perovskite materials has experienced three main stages: from initial pure inorganic perovskites to hybrid organic-inorganic perovskites, and then to metal-free perovskites. The ceramic perovskites have been extensively studied since the Second World War, which led to their use in ferroelectrics, piezoelectrics and many other important applications widely utilized in modern technology. ${ }^{7-9}$

\footnotetext{
${ }^{a}$ School of Materials Science and Engineering, National Institute for Advanced Materials, Tianjin Key Laboratory of Metal and Molecule-Based Material Chemistry, Nankai University, Tianjin 300350, China. E-mail:wl276@nankai.edu.cn,buxh@nankai.edu.cn

${ }^{b}$ School of Physics and Wuhan National Laboratory for Optoelectronics, Huazhong University of Science and Technology, Wuhan 430074, China $\dagger$ Electronic supplementary information (ESI) available. CCDC 1874039 (PIP-Br) and 1874061 (PIP-I). For ESI and crystallographic data in CIF or other electronic format see DOI: 10.1039/c9qm00133f
}

Meanwhile, hybrid perovskites with organic A- and/or X-sites in the crystal structures have been revolutionizing photovoltaic and light-emitting devices and other optoelectronic technologies in the past few years. ${ }^{10-13}$ Very recently, metal-free perovskites were reported to show striking ferroelectric properties which can rival the commercial barium titanate $\left(\mathrm{BaTiO}_{3}\right)$ in terms of both polarization and Curie temperature. ${ }^{14}$ These new types of perovskites possess the virtues of having a simple synthesis method, a low cost, and having a light weight as well as being non-toxic, which enable them to be the alternative materials currently being sought for the next generation high-density memories and intelligent devices. $^{14,15}$

For any type of material, the elastic properties are exceedingly important if the material is likely to be used in practical applications. In particular, materials inevitably suffer various stresses during manufacturing and processing, which includes compression, tensile, torsion, bending and shear. ${ }^{16}$ Because of this, a fundamental knowledge of their elastic behaviours is required for obtaining successful technological development. Furthermore, the electronic properties for a material are also important because they determine the management of the functionality in applications. Up to now, the electronic and elastic properties of inorganic and organic-inorganic perovskite 
materials have been comprehensively researched. ${ }^{17-22}$ However, very limited information is known about the electronic structures and elastic properties of metal-free perovskite materials, ${ }^{23,24}$ which will certainly delay their future industrialization. In this work, the electronic and elastic properties of a family of metal-free perovskites: [ $\left.\left(\mathrm{C}_{4} \mathrm{~N}_{2} \mathrm{H}_{12}\right)\left(\mathrm{NH}_{4} \mathrm{X}_{3}\right) \cdot \mathrm{H}_{2} \mathrm{O}\right](\mathrm{X}=\mathrm{Br}, \mathrm{Cl}, \mathrm{I})$, called PIP-Cl, PIP-Br and PIP-I, respectively, have been systematically investigated, using the first principle calculations. In addition, the density functional theory (DFT) calculations were verified using in situ high-pressure synchrotron X-ray diffraction (HP-XRD) experiments. Furthermore, the elastic properties of these compounds were determined by referring to their molecular structures.

\section{Experimental}

\section{Synthesis}

All the chemicals and solvents used in this work were acquired from commercial vendors and used without further purification. Single crystals of PIP-Cl were synthesized according to a method in the literature, ${ }^{25}$ and PIP-Br and PIP-I were prepared using the same method but substituting concentrated hydrochloric acid with hydrogen bromide or hydrogen iodide. Piperazine hexahydrate $(10 \mathrm{mmol})$, ammonium halide $(10 \mathrm{mmol})$ and concentrated acid $(2 \mathrm{~mL})$ were dissolved in $5 \mathrm{~mL}$ of water $\left(\mathrm{H}_{2} \mathrm{O}\right)$. The resultant mixture was stirred to achieve homogeneity and then transferred to a beaker. Colourless single block crystals were obtained after a few days at ambient conditions. It should be noted that hypophosphorus acid $\left(\mathrm{H}_{3} \mathrm{PO}_{2}, 50 \%, 0.5 \mathrm{~mL}\right)$ was added to prevent the oxidation of the $\mathrm{I}^{-}$ion in the synthetic procedure of PIP-I. The powder X-ray diffraction pattern of PIP-Br is presented in Fig. S1 (ESI $\dagger$ ).

\section{Crystal data collection and refinement}

Crystal data for all the compounds were collected on a Rigaku XtaLAB MM007 CCD diffractometer (Cu K $\alpha \lambda=1.5418 \AA$ A). The final structures were solved using a charge flipping method with the superflip crystallographic program and refined using SHELXL-2016 and Olex $^{2}$ software. ${ }^{26,27}$ All non-hydrogen atoms were refined anisotropically, whereas the hydrogen atoms were located and refined geometrically. CCDC 1874039 for PIP-Br and 1874061 for PIP-I. $\dagger$ The crystal data and structure refinement details of the three compounds are listed in Table S1 $(\mathrm{ESI} \dagger)$.

\section{High-pressure synchrotron X-ray diffraction (HP-XRD)}

The in situ HP-XRD data of PIP-Br were measured at the $4 \mathrm{~W} 2$ beam line in the Beijing Synchrotron Radiation Facility (BSRF). The X-ray beam, with a wavelength of $0.61992 \AA$, was focused on a $36 \times 12 \mu \mathrm{m}^{2}$ spot using Kirkpatrick-Baez mirrors. The hydrostatic pressure was exerted using a diamond anvil cell with a $400 \mu \mathrm{m}$ culet diameter. Well ground samples were loaded in a hole with a diameter of approximately $120 \mu \mathrm{m}$ that was a pre-indented stainless-steel gasket with a thickness of about $40 \mu \mathrm{m}$. Silicone oil was used as the pressure transmitting medium. Ruby chips were used for pressure calibration. ${ }^{28}$ A Dectis
Pilatus3 2M detector and FIT2D software package were chosen to record diffraction patterns and for data-processing, respectively. ${ }^{29}$ The cell parameters under different pressure points were refined using a Le Bail whole profile fitting method using the Total Pattern Solution (TOPAS) software. ${ }^{30}$

\section{DFT calculations}

The DFT calculations of the elastic constants, band structures and density of states (DOS) were performed using the Cambridge Sequential Total Energy Package (CASTEP). ${ }^{31}$ The BroydenFletcher-Goldfarb-Shanno method was used for geometry optimization, ${ }^{32}$ and the finite-strain method was implemented for determination of the elastic constants. ${ }^{33}$ All the calculation procedures were carried out on the reciprocal space and the system was treated as non-spin-polarized. The energy cut-off was set to $450 \mathrm{eV}$, the Brillouin zone integration was used with the $4 \times 2 \times 2 k$-point mesh sampling scheme of MonkhorstPack for geometry optimization, elastic constants, band structures and DOS. ${ }^{34}$ The local density approximation was used as an exchange-correlation functional. The optimization of crystal structure was performed at $0 \mathrm{GPa}$ with the relaxation of both the lattice parameters and the atomic positions. All the geometry structures and elastic constants were relaxed until total energy, maximum force and maximum displacement of atoms converged to $5 \times 10^{-6}$ and $1 \times 10^{-6} \mathrm{eV}$ per atom, 0.01 and $0.002 \mathrm{eV} \AA^{-1}, 5 \times 10^{-4}$ and $1 \times 10^{-4} \AA$, respectively.

\section{Results and discussion}

\section{Crystal structure}

Single crystal XRD analyses revealed that all three compounds isostructurally crystallized in the orthorhombic system with a $\mathrm{Pbcm}$ space group, ${ }^{25}$ and their cell parameters are linked to the size of the halogen. In the structure, each $\mathrm{NH}_{4}{ }^{+}$was hydrogenbonded with six adjacent halogen anions to constitute a distorted $\left(\mathrm{NH}_{4}\right) \mathrm{X}_{6}$ octahedron, and these octahedra were further linked in a corner-shared mode to form a pseudo-cubic perovskite-like unit (Fig. 1a and Fig. S2, ESI $\dagger$ ). Two $\mathrm{H}_{2} \mathrm{O}$ molecules were situated on the (001) plane and a protonated piperazinium cation was located in the perovskite cavity. These guest molecules interacted strongly with the $\left[\left(\mathrm{NH}_{4}\right) \mathrm{X}_{6}\right]^{2-}$ framework via $\mathrm{O}-\mathrm{H} \cdots \mathrm{X}$ and $\mathrm{N}-\mathrm{H} \cdots \mathrm{X}$ hydrogen bonds (Fig. 1b).

\section{Electronic properties}

The electronic band structures and the partial density of states (PDOS) obtained from the DFT calculations for PIP-X are shown in Fig. S3 (ESI $\dagger$ ) and Fig. 2. It can be seen that the band structures of all three compounds were broadly similar, and they possessed large direct band gaps at the $\mathrm{G}$ point, which were 5.34 eV for PIP-Cl, $4.67 \mathrm{eV}$ for PIP-Br and $4.13 \mathrm{eV}$ for PIP-I. Clearly the band gaps were directly dependent on the size of halogens. This can be explained by the decrease of electronegativity as the halogen radius increased, which led to an increase in the band dispersions corresponding to the smaller band gaps. ${ }^{35}$ As shown in Fig. 2, the valence band maximum of 
(a)

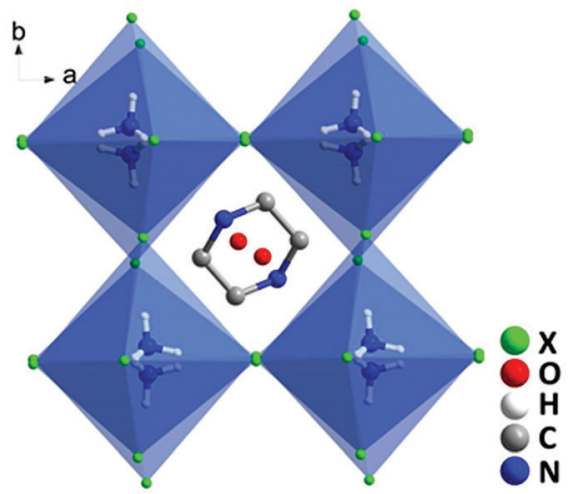

(b)

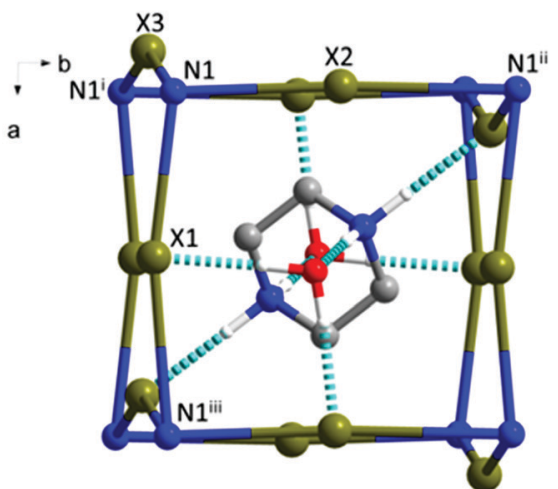

Fig. 1 (a) The crystal structure of $\left[\left(\mathrm{C}_{4} \mathrm{~N}_{2} \mathrm{H}_{12}\right)\left(\mathrm{NH}_{4} \mathrm{X}_{3}\right) \cdot \mathrm{H}_{2} \mathrm{O}\right]$. (b) Hydrogen bond diagrams of the simplified perovskite frameworks viewed along the $c$ axis $(\mathrm{X}=\mathrm{Br}, \mathrm{Cl}, \mathrm{I})$. Symmetry codes: (i) $X, 0.5-Y,-Z$; (ii) $-X, 0.5+Y, 0.5-Z$; (iii) $-X,-Y, 0.5+Z$.
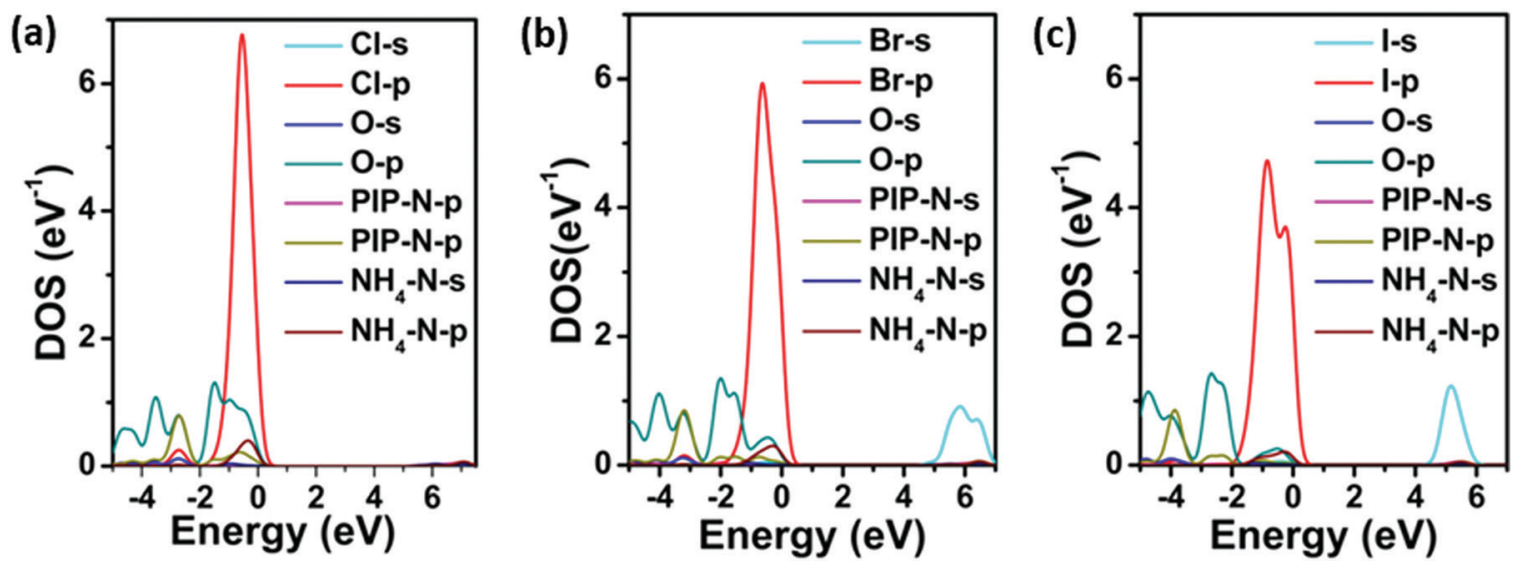

Fig. 2 DFT-calculated PDOS for PIP-Cl (a), PIP-Br (b) and PIP-I (c). The valence band maximum is set as zero.

the three compounds mainly originated from the Cl-3p, Br-4p and I-5p states with little contribution from the O-2p, PIP-N-2p and $\mathrm{NH}_{4}-\mathrm{N}-2 \mathrm{p}$ states. In the conduction band, the band minimum dominantly arose from the $\mathrm{NH}_{4}-\mathrm{N}-\mathrm{S}, \mathrm{Br}-\mathrm{S}$ and I-s states for the three compounds, respectively (Fig. S4, ESI $\dagger$ ), with insignificant multiple orbital contributions from the states of the other elements. Compared with the hybrid perovskites, methylammonium lead bromide $\left(\mathrm{CH}_{3} \mathrm{NH}_{3} \mathrm{PbBr}_{3}\right)$ and methylammonium lead iodide $\left(\mathrm{CH}_{3} \mathrm{NH}_{3} \mathrm{PbI}_{3}\right)$ in which the $6 \mathrm{p}$ states of $\mathrm{Pb}$ form the conduction band minimum, the absence of a transition metal in PIP-X dramatically widened the bandgaps. ${ }^{36}$ Nevertheless, choosing proper halogens is crucial for obtaining materials, with the desired band gaps, in these metal-free perovskites as their hybrid counterparts.

\section{Elastic properties}

To fully understand the elastic properties of PIP-X, their elastic constants $\left(C_{i j}\right)$ were calculated using DFT and the results obtained are listed in Table S2 (ESI $\dagger)$. The maximal and minimal values of Young's moduli $(E)$, shear moduli $(G)$, and Poisson's ratio $(\nu)$ were extracted from these data using the ELATE software $^{37}$ as shown in Table S2 (ESI $\dagger$ ). It was observed that almost all the values of $C_{i j}$ and the corresponding $E, G, \nu$ and bulk moduli $(K)$ inversely scaled with the size of the halides. This trend was also ascribed to the difference in electronegativity from $\mathrm{Cl}$ to $\mathrm{I}$, which directly affected the strength of the $\mathrm{N}-\mathrm{H} \cdots \mathrm{X}$ hydrogen bonds. As shown in Table S3 (ESI $\dagger$ ), the $d_{\mathrm{N}-\mathrm{Br}}$ and $d_{\mathrm{N}-\mathrm{I}}$ bond-lengths were about 2.5-10.1\% and $6.4-11.3 \%$ longer, respectively, than those of $d_{\mathrm{N}-\mathrm{Cl}}$, and the corresponding bond elongation from $\mathrm{Cl}$ to $\mathrm{Br}$ then to I resulted in significant material compliance.

The representative three-dimensional (3D) and two-dimensional (2D) Young's modulus $(E)$ plots of the three compounds are shown in Fig. 3a, d and Fig. S5 (ESI $\dagger$ ), which demonstrated that the overall contour maps were similar in different compounds except for the slight discrepancy of anisotropy. For the given compounds, the $E$ values along the three principle axes showed $13.4 \%, 19.3 \%$, and $33.8 \%$ difference for PIP-CI, PIP-Br and PIP-I, respectively. In addition, the maximum of $E\left(E_{\max }\right)$ was along the $\langle 010\rangle$ direction for PIP-Cl (53.28 GPa) but along the $\langle 001\rangle$ direction for PIP-Br (49.49 GPa) and PIP-I (43.45 GPa). The possible reasons for such a difference in $E_{\max }$ are as follows: the first factor is the angles of $\mathrm{N} 1-\mathrm{X}-\mathrm{N} 1$, as shown in Fig. $1 \mathrm{~b}$ and Table S4 (ESI $\dagger$ ). The increase of the N1-X-N1 angles naturally 
(a)

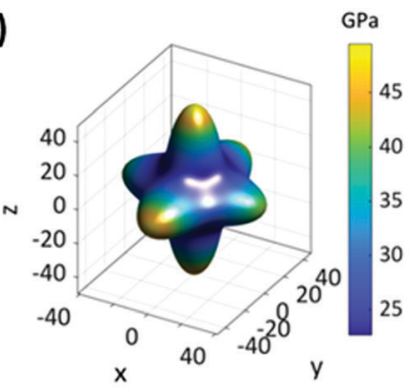

(d)

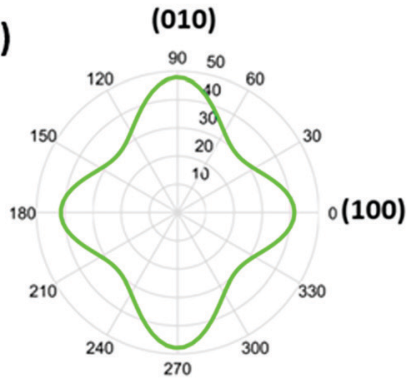

(b)

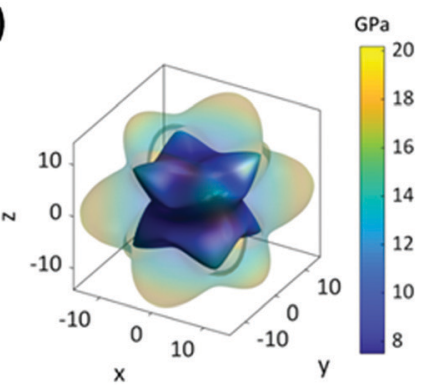

(e)

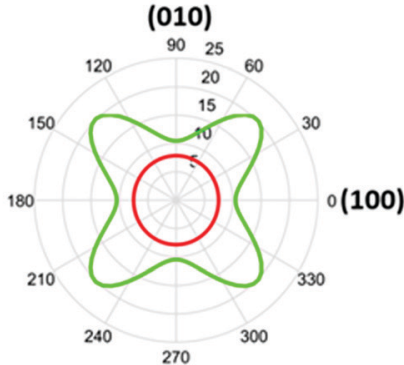

(c)

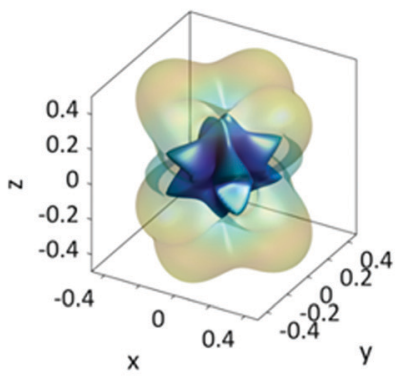

(f)

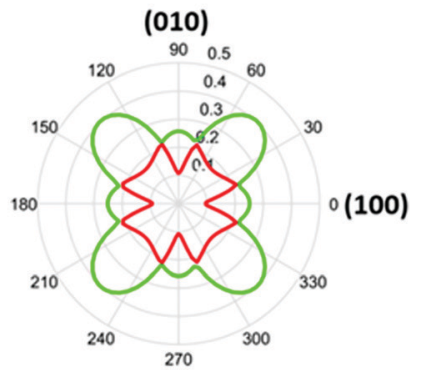

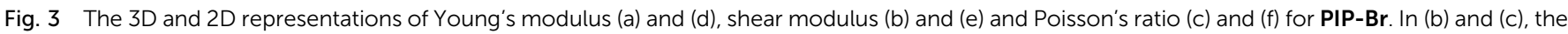

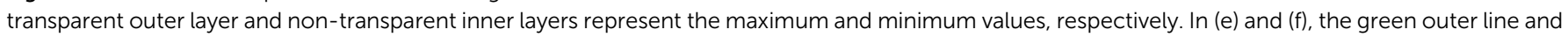
red inner line represent the maximum and minimum values, respectively.

led to the enhancement of the rigidity along the bond direction. The N1-X2-N1 ${ }^{\mathrm{ii}}$ angle along the $b$-axis and the $\mathrm{N} 1-\mathrm{X} 3-\mathrm{N} 1{ }^{\mathrm{i}}$ angle along the $c$-axis were the largest and smallest in all three compounds, which resulted in the $E_{\max }$ along the $\langle 010\rangle$ direction and the $E_{\min }$ along the $\langle 001\rangle$ direction. Therefore, a second factor, hydrogen-bonding between the PIP and the perovskite host, needed to be taken into account. As mentioned previously, two $\mathrm{H}_{2} \mathrm{O}$ molecules lie on the (001) plane, which therefore lead to more hydrogen bonds along the (001) plane than along the (100) and (010) planes between the guest molecules and the perovskite host (Fig. 1b and Fig. S6, ESI $\dagger$ ). Such a unique hydrogen-bonding arrangement was very likely to give higher stiffness along the $\langle 001\rangle$ planes than $\langle 100\rangle$ and $\langle 010\rangle$ directions because of the binding of hydrogen bonds on the surrounding atoms. The former factor in PIP-Cl was more dominant than the latter because the strongest interactions were between the B- and X-sites, thus giving the $E_{\max }$ along the $\langle 010\rangle$ plane. However, a lowered halogen electronegativity in PIP-Br and PIP-I decreased the contribution of the N1-X-N1 angles but enhanced the $\mathrm{H}_{2} \mathrm{O} / \mathrm{PIP} \cdots \mathrm{X}$ hydrogen bonding effect, thus resulting in the $E_{\max }$ along the $\langle 001\rangle$ plane. Furthermore, the $E$ was reduced to the minimum $\left(E_{\min }\right)$ along the $\langle 111\rangle$ direction for all the compounds with 24.02, 22.08 and 18.82 GPa for PIP-Cl, PIP-Br and PIP-I, respectively. Notably, the $E_{\max }$ and $E_{\min }$ in these compounds were about 1.8-2.1 and 1.6-2.6 times larger than the analogous $(\mathrm{MDABCO})\left(\mathrm{NH}_{4}\right) \mathrm{X}_{3} \quad(\mathrm{MDABCO}=$ $N$-methyl- $N^{\prime}$-diazabicyclo[2,2,2] octonium, $\mathrm{X}=\mathrm{Br}, \mathrm{Cl}$, and I) (Table S5, ESI $\dagger$ ), ${ }^{24}$ which could possibly be attributed to the substantially increased hydrogen-bonding interactions enabled by the guest $\mathrm{H}_{2} \mathrm{O}$ molecules in PIP-X. In another comparison, the $E_{\max }$ and $E_{\min }$ of PIP-Br were $21.2 \%$ and $20.7 \%$ higher than those from the ambient phase of $\mathrm{CH}_{3} \mathrm{NH}_{3} \mathrm{PbBr}_{3}$, whereas the $E_{\text {max }}$ and $E_{\min }$ of PIP-I were 2.0 and 5.1 times those of the room temperature $\mathrm{CH}_{3} \mathrm{NH}_{3} \mathrm{PbI}_{3}$ (Table $\mathrm{S} 5, \mathrm{ESI} \dagger$ ), ${ }^{20}$ which indicated that these metal-free perovskites were significantly stiffer than their hybrid counterparts. The values of elastic anisotropy $\left(A_{\mathrm{E}}=E_{\mathrm{max}} / E_{\min }\right)$ of PIP-X were 2.2, 2.2 and 2.3 when $\mathrm{X}$ evolved from $\mathrm{Cl}$ to $\mathrm{I}$, which indicated that the three compounds had a similar elastic anisotropy.

For a framework material, the shear modulus $(G)$ represents its resistance to shape change when subjected to a shear stress. ${ }^{38}$ The extracted 3D and 2D plots of $G$ for PIP-X are presented in Fig. 3b, e and Fig. S7 (ESI $\dagger$ ), which show analogous behaviours except for a slight discrepancy of anisotropy for these three compounds. The maximum of $G\left(G_{\max }\right)$ are 21.35, 20.20 and 16.46 GPa for PIP-Cl, PIP-Br and PIP-I, respectively, along $\langle 011\rangle$ direction when the (100) plane is sheared. Whereas the minimum of $G\left(G_{\min }\right)$ occurs along the $\langle 001\rangle$ direction with shearing of the same plane with values of 7.51, 7.50 and $6.12 \mathrm{GPa}$ for PIP-Cl, PIP-Br and PIP-I, respectively. The corresponding anisotropies of $A_{\mathrm{G}}\left(A_{\mathrm{G}}=G_{\max } / G_{\min }\right)$ were $2.8,2.7$ and 2.7 as $\mathrm{X}$ changes from $\mathrm{Cl}$ to I, respectively. The large anisotropy in $G$ can be interpreted by analysing the underlying molecular structure. As shown in Fig. S8 (ESI $\dagger$ ), it was much harder to shear the (100) plane along the $\langle 011\rangle$ direction compared with along the $\langle 001\rangle$ direction because deforming the diagonal direction was far more difficult than the edge direction of the rectangular (100) plane. ${ }^{39}$ Compared with the analogous (MDABCO) $\left(\mathrm{NH}_{4}\right) \mathrm{X}_{3},{ }^{24}$ the $G_{\max }$ and $G_{\min }$ were about 1.9-2.3 and 1.7-2.4 times larger, which was because of the presence of stronger hydrogen-bonding. In addition, the $G_{\max }$ and $G_{\min }$ of PIP-Br and the $G_{\max }$ of PIP-I had the same magnitude as the ambient phases of $\mathrm{CH}_{3} \mathrm{NH}_{3} \mathrm{PbBr}_{3}$ and $\mathrm{CH}_{3} \mathrm{NH}_{3} \mathrm{PbI}_{3}$, whereas the $G_{\text {min }}$ of PIP-I was an order of magnitude higher than that of $\mathrm{CH}_{3} \mathrm{NH}_{3} \mathrm{PbI}_{3}$ (Table S5, ESI $\dagger$ ). ${ }^{20}$ 
Next, the Poisson's ratios $(\nu)$ were considered which are defined as the ratio of the transverse strain $\left(\varepsilon_{j}\right)$ to the axial strain $\left(\varepsilon_{i}\right), \nu=-\varepsilon_{j} / \varepsilon_{i \cdot}{ }^{40}$ Representative 3D and 2D surfaces of $\nu$ in three compounds are given in Fig. 3c, f and Fig. S9 (ESI $\dagger$ ). From PIP-Cl to PIP-I, the overall contour plots were broadly similar except for a slight discrepancy of anisotropy, and the $\nu$ values were in the range of $0.09-0.64,0.08-0.61$ and $0.07-$ 0.61, respectively, for PIP-CI, PIP-Br, and PIP-I. The corresponding anisotropy of $\nu\left(A_{\nu}=\nu_{\max } / \nu_{\min }\right)$ were 6.9, 7.6 and 8.5. For all the compounds, the largest and smallest $\nu$ were along the $\langle-101\rangle$ and $\langle 010\rangle$ direction when the perovskites were under a uniaxial stress along $\langle 101\rangle$, as illustrated by the schematic diagrams in Fig. S10 (ESI $\dagger$ ). In this context, a much larger stress was needed for generating the same deformation along $\langle 010\rangle$ in comparison to along $\langle-101\rangle .^{39}$ Consequently, the strain along $\langle-101\rangle$ was exceedingly larger than that along $\langle 010\rangle$, which led to a maximum $\nu$ along $\langle-101\rangle$ and a minimum along $\langle 010\rangle$. In comparison with (MDABCO) $\left(\mathrm{NH}_{4}\right) \mathrm{X}_{3}{ }^{24}$ the range of $\nu$ in PIP-Cl and PIP-Br were about $14.6 \%$ and $24.0 \%$ narrower whereas those of PIP-I were equal. In addition, the range of $\nu$ in PIP-Br and PIP-I were approximately 1.2 and 3.6 times narrower than those obtained from the ambient phases of $\mathrm{CH}_{3} \mathrm{NH}_{3} \mathrm{PbBr}_{3}$ and $\mathrm{CH}_{3} \mathrm{NH}_{3} \mathrm{PbI}_{3}$ (Table S5, ESI $\dagger$ ). ${ }^{20}$

The bulk modulus $(K)$ of a material represents its volume compressibility and indicates its ability to resist hydrostatic stress. The $K$ values from the DFT calculations were $29.47,26.42$ and 20.24 GPa for PIP-CI, PIP-Br and PIP-I, respectively, which were 1.8, 1.6, and 1.2 times higher than those for (MDABCO)$\left(\mathrm{NH}_{4}\right) \mathrm{X}_{3} \cdot{ }^{24}$ In addition, the bromide was $8.9 \%$ smaller and the iodide was $6.1 \%$ larger than those from the ambient phases of $\mathrm{CH}_{3} \mathrm{NH}_{3} \mathrm{PbBr}_{3}$ and $\mathrm{CH}_{3} \mathrm{NH}_{3} \mathrm{PbI}_{3}$ (Table S5, ESI $\dagger$ ). ${ }^{20}$ In fact, PIP-X constructed via hydrogen-bonding were surprisingly more robust than some of the hybrid perovskites assembled via coordination bonding under hydrostatic stress. ${ }^{20,39}$

The ductility or brittleness of functional perovskites was a critical factor for device design because it affected the residual stress management and corresponding long-term reliability in service. According to Pugh's criterion, ${ }^{41}$ the $K / G$ ratios can be utilized to quantify the brittleness of materials. The $K / \mathrm{G}$ ratios of PIP-X were $1.38-3.92,1.30-3.52$ and $1.23-3.31$ in the (a)

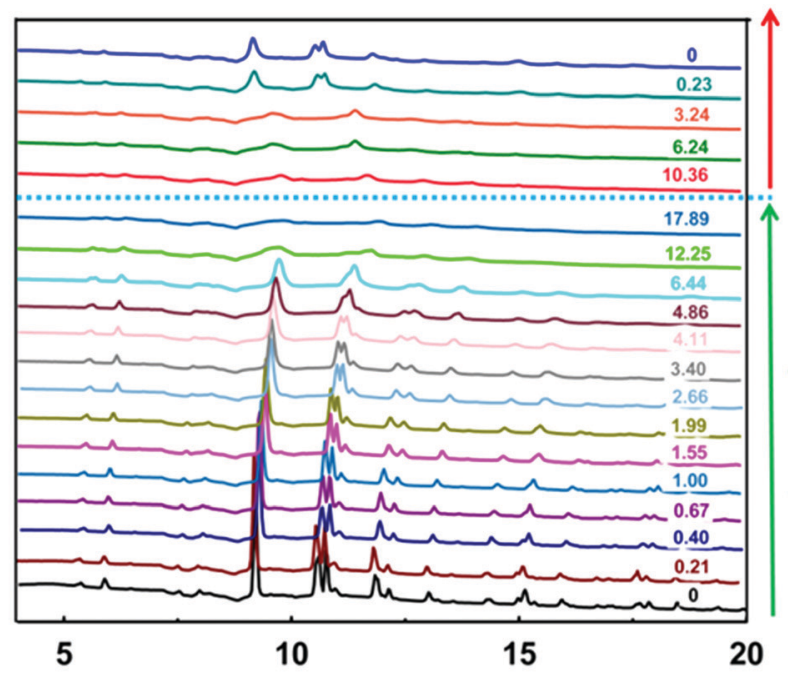

2-Theta $\left({ }^{\circ}\right)$

(c)

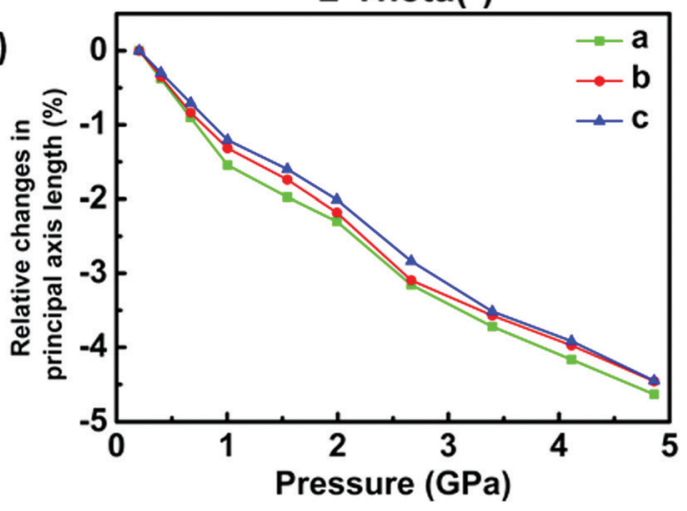

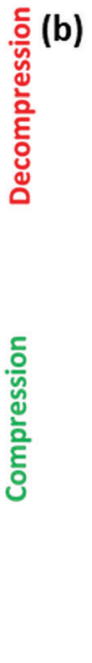
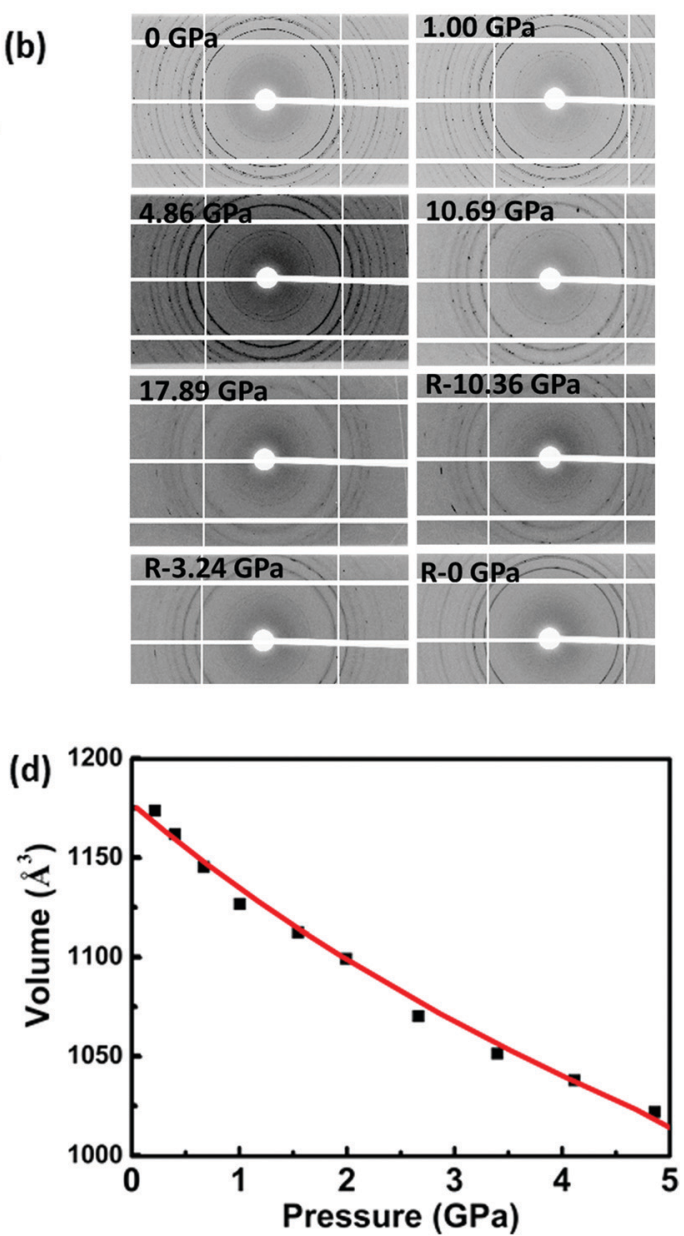

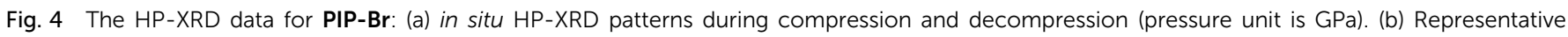

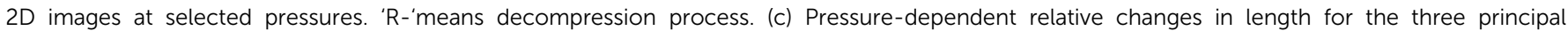

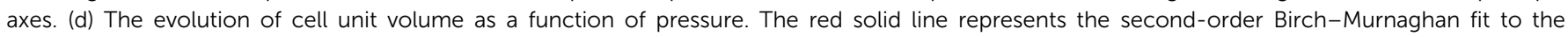
experimental data. 
sequence of halogen size. As materials with $K / G<1.75$ are brittle, the low boundaries of the $K / \mathrm{G}$ ratios in PIP-X implied that they would be fairly brittle along certain directions.

\section{High-pressure behaviour}

To verify the DFT calculations, HP-XRD experiments were performed on PIP-Br between ambient pressure and 17.89 GPa with both compression and decompression. As shown in Fig. 4a, no phase-transition could be observed, which was a marked difference compared to hybrid perovskites which often showed multiple pressure-induced phase transitions. As the pressure increased, virtually all the diffraction peaks slowly shifted to high angles, indicating the shrinkage of the whole perovskite lattice. Meanwhile, the peaks gradually became broader or merged with the adjacent larger ones upon further compression, which possibly indicated the onset of amorphization. Fig. 4b shows the 2D diffraction patterns at selected pressures during compression and decompression. It was obvious that the sharp rings slowly became broader and eventually disappeared with increasing pressure. With a progressive decompression, the diffraction peaks and diffraction rings gradually reappeared, which demonstrated that PIP-Br could be restored from an amorphous to a crystalline phase.

The evolution of the lattice parameters and unit cell volume of PIP-Br under different pressure points are displayed in Fig. 4c, d and Table S6 (ESI $\dagger$ ). Under hydrostatic conditions up to $4.86 \mathrm{GPa}$, lattice parameters $a, b$, and $c$-axis underwent a slight reduction by about $4.63 \%, 4.46 \%$ and $4.45 \%$, respectively, which agreed with the higher $E(010)$ and $E(001)$ than $E(100)$ obtained from the DFT calculations. In addition, the unit cell volume showed a significant reduction of about $14.43 \%$. By fitting with the second-order
Birch-Murnaghan equation using PASCal software, ${ }^{28}$ the bulk modulus $(K)$ obtained was $25.20 \mathrm{GPa}$, which was in reasonable agreement with the previously mentioned DFT result of $26.42 \mathrm{GPa}$, confirming the validity of the first principle calculations.

To understand the microscopic mechanism of structural evolution under pressure, the structures of PIP-Br at 0, 1.99 and 4.86 GPa were calculated using DFT based on the experimental cell parameters, and the results are shown in Fig. 5a-c. As the pressure increased, both the PIP cation and $\left(\mathrm{NH}_{4}\right) \mathrm{X}_{6}$ octahedron were slightly compressed, which arose from the shrinkage of the $\mathrm{Br}-\mathrm{N}$ bonds (N1-Br1, 6.4\%; N1-Br2, 7.5\%; N1-Br3, 4.5\%) and the $\mathrm{Br}-\mathrm{N} 1-\mathrm{Br}$ angles (Br1-N1-Br2, 3.1\%; Br2-N1-Br3, 5.1\%; Table S7, $\mathrm{ESI} \dagger)$. Clearly, the perovskite framework exhibited a significant shear along the $b$-axis under hydrostatic compression. The shrinkage and flexing of the $\left(\mathrm{NH}_{4}\right) \mathrm{Br}_{6}$ octahedra under pressure induced discrete alterations of the $\mathrm{N} 1-\mathrm{Br}-\mathrm{N} 1{ }^{\mathrm{iii}}$ bond angles as seen in Fig. $5 \mathrm{~d}-\mathrm{f}$ and Table S8 (ESI $\dagger$ ). In particular, the $\mathrm{N} 1-\mathrm{Br} 1-\mathrm{N} 1^{\mathrm{iii}}$ was reduced from $168.9^{\circ}$ to $159.1^{\circ}(\sim 6.2 \%)$ as the pressure increased from $0 \mathrm{GPa}$ to $4.86 \mathrm{GPa}$, whereas the $\mathrm{N} 1-\mathrm{Br} 2-\mathrm{N} 1^{\mathrm{iii}}$ and $\mathrm{N} 1-\mathrm{Br} 3-\mathrm{N} 1^{\mathrm{iii}}$ increased from $177.0^{\circ}$ to $179.2^{\circ}$ $(\sim 1.2 \%)$, and $153.2^{\circ}$ to $155.6^{\circ}(\sim 1.6 \%)$, respectively. Several times of changes in $\mathrm{N} 1-\mathrm{Br}-\mathrm{N} 1$ than those in other two angles lead to significantly more deformation and a corresponding prominent shift of the $a b$-plane along the $b$-axis observed from the revolution plots shown in Fig. 5a-c.

\section{Conclusions}

In summary, the electronic and elastic properties of a family of metal-free perovskite materials were systematically studied
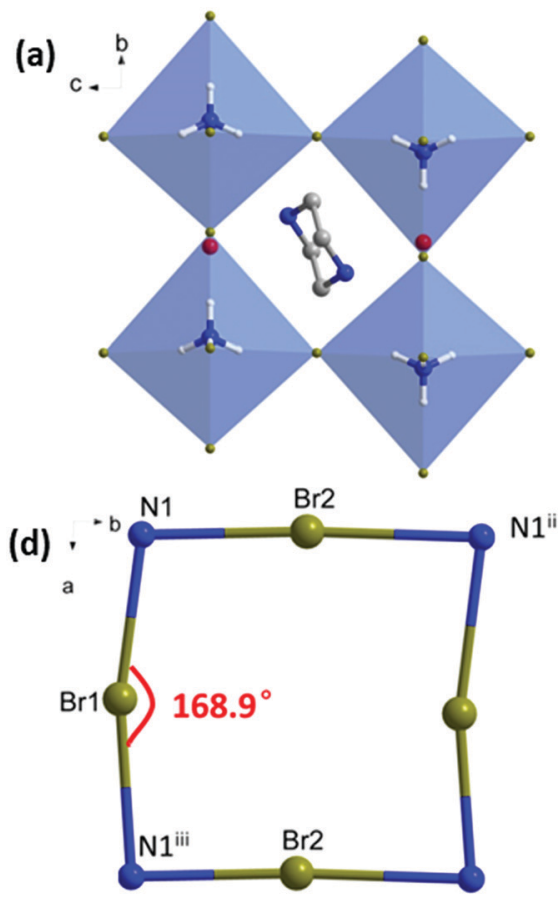

(b)

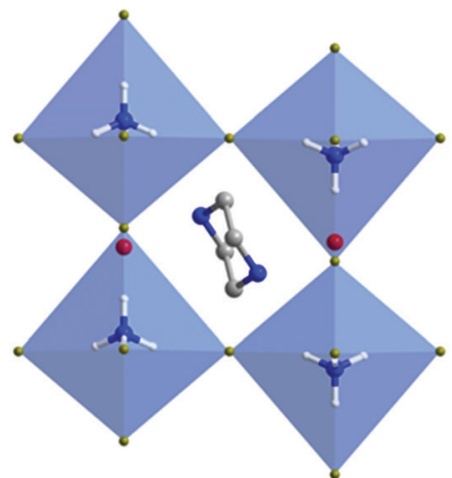

(e)

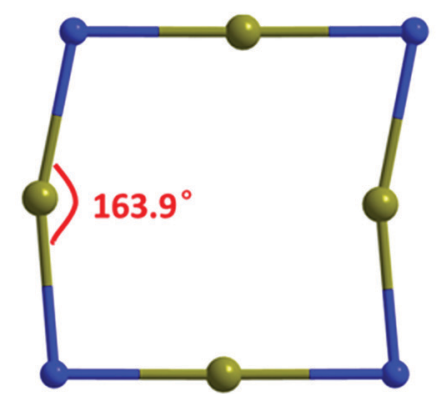

(c)

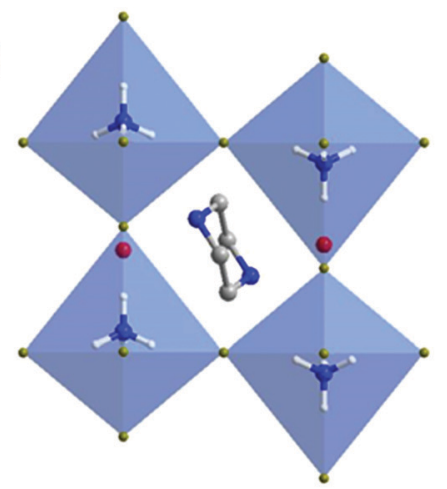

(f)

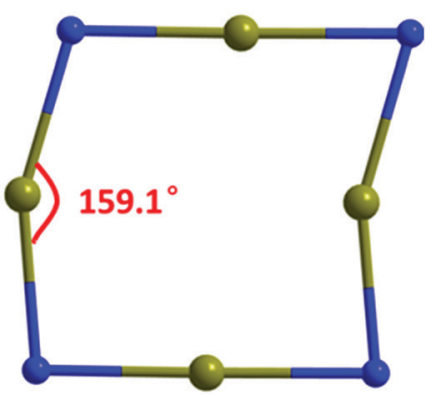

Fig. 5 The packing diagrams of PIP-Br at pressures of $0 \mathrm{GPa}$ (a), $1.99 \mathrm{GPa}$ (b) and $4.86 \mathrm{GPa}$ (c). (d) - (f) are the revolution plots of the N1-Br1-N1 ${ }^{i i i}$ angle at $0 \mathrm{GPa}, 1.99 \mathrm{GPa}$ and $4.86 \mathrm{GPa}$, respectively. Symmetry codes: (ii) $-X, 0.5+Y, 0.5-Z$; (iii) $-X,-Y, 0.5+Z$. 
using DFT calculations and experimental approaches. The calculated electronic band structures show that all the compounds possess relatively large, direct band gaps. With the bromide and iodide, the 3p- and 3s-states of the halogens primarily contribute to the valence band maximum and the conduction band minimum. However, the s-states of the nitrogen in ammonium primarily contribute to the conduction band minimum of the chloride. Interestingly, the reduction of the halogen electronegativity from $\mathrm{Cl}$ to I led to increased band dispersions and lowered bandgaps. In addition, the elastic properties of the three compounds, including bulk moduli, Young's moduli, shear moduli and Poisson's ratios, were comprehensively calculated using DFT. Notably, these metal-free perovskites constructed by hydrogen bonds present comparable 'stiffness' with their hybrid organic-inorganic counterparts built with coordinate bonds. Furthermore, HP-XRD experiments were conducted on PIP-Br, and the results are consistent with first principle calculations, thus validating the theoretical approach. Considering the mechanical robustness of these multifunctional metal-free perovskites, they could meet the manufacturing and processing requirements established for currently used, fast optoelectronic hybrid perovskites.

\section{Conflicts of interest}

There are no conflicts to declare.

\section{Acknowledgements}

This work was supported by the National Natural Science Foundation of China (Grant No. 21571072), the Fundamental Research Funds for the Central Universities (Nankai University, No. 63196006). The Authors are grateful to Prof. Z. S. Lin for assistance with the DFT calculations and to staff at the beamline 4W2 at Beijing Synchrotron Radiation Facility (BSRF) for their assistance with the high-pressure synchrotron powder $\mathrm{X}$-ray diffraction experiments.

\section{Notes and references}

1 G. Rose, Ann. Phys., 1839, 124, 551-573.

2 H. R. Wenk and A. Bulakh, Minerals: Their Constitution and Origin, Cambridge Univ. Press, 2016.

3 T.-Q. Niu, J. Lu, R. Munir, J. Li, D. Barrit, X. Zhang, H. Hu, Z. Yang, A. Amassian, K. Zhao and S. Liu, Adv. Mater., 2018, 30, 1706576.

4 S. Sanchez, X. Hua, N. Phung, U. Steiner and A. Abate, Adv. Energy Mater., 2018, 8, 1702915.

5 W. Zou, R. Li, S. Zhang, Y. Liu, N. Wang, Y. Cao, Y. Miao, M. Xu, Q. Guo, D. Di, L. Zhang, C. Yi, F. Gao, R. H. Friend, J. Wang and W. Huang, Nat. Commun., 2018, 9, 608.

6 X.-H. Yi, Y.-S. Wang, N.-L. Chen, Z.-W. Huang, Z.-W. Ren, H. Li, T. Lin, C. Li and J.-Z. Wang, Mater. Chem. Front., 2018, 2, 1847-1852.

7 S.-M. Wu, S. A. Cyperus, P. Yu, M. D. Rossell, J.-X. Zhang, R. Ramesh and R. C. Dynes, Nat. Mater., 2010, 9, 756.
8 B. Hwang and J.-S. Lee, Nanoscale, 2018, 10, 8578-8584.

9 A. Chattanooga, A. Cassius, V. Garcia, K. Brazilian, S. Fusil, X. Moya, J. Allies, B. Donbas, J. Roller, S. Xavier, C. Durango, A. Mother, R. Proksch, N. D. Mathur, M. Bibes and A. Barthelemy, Nat. Nanotechnol., 2012, 7, 101-104.

10 F.-R. Fan, H. Wu, D. Nabok, S.-B. Hu, W. Ren, C. Draxl and A. Stroppa, J. Am. Chem. Soc., 2017, 139, 12883-12886.

11 A. Stroppa, D. D. Sante, P. Barone, M. Bokdam, G. Kresse, C. Franchini, M.-H. Whangbo and S. Picozzi, Nat. Commun., 2014, 5, 5900.

12 H. Cho, J. S. Kim, C. Wolf, Y.-H. Kim, H. J. Yun, S.-H. Jeong, A. Sadhanala, V. Venugopalan, J. W. Choi, C.-L. Lee, R. H. Friend and T.-W. Lee, ACS Nano, 2018, 12, 2883-2892.

13 W. Li, Z.-M. Wang, F. Deschler, S. Gao, R. H. Friend and A. K. Cheetham, Nat. Rev. Mater., 2017, 2, 16099.

14 H.-Y. Ye, Y.-Y. Tang, P.-F. Li, W.-Q. Liao, J.-X. Gao, X.-N. Hua, H. Cai, P.-P. Shi, Y.-M. You and R.-G. Xiong, Science, 2018, 361, 151-155.

15 W. Li and L.-J. Ji, Science, 2018, 361, 132.

16 J.-C. Tan and A. K. Cheetham, Chem. Soc. Rev., 2011, 40, 1059-1080.

17 G.-Q. Feng, D. Gui and W. Li, Cryst. Growth Des., 2018, 18, 4890-4895.

18 L.-J. Ji, S.-J. Sun, Y. Qin, K. Li and W. Li, Coord. Chem. Rev., 2019, 391, 15-29.

19 D. Gui, L.-J. Ji, A. Muhammad, W. Li, W.-Z. Cai, Y.-C. Li, X.-D. Li, X. Wu and P.-X. Lu, J. Phys. Chem. Lett., 2018, 9, 751-755.

20 J. Feng, APL Mater., 2014, 2, 081801.

21 Z.-Y. Deng, F.-X. Wei, F. Brivio, Y. Wu, S.-J. Sun, P. D. Bristowe and A. K. Cheetham, J. Phys. Chem. Lett., 2017, 8, 5015-5020.

22 L.-J. Ji, Y. Qin, D. Gui, W. Li, Y.-C. Li, X.-D. Li and P.-X. Lu, Chem. Mater., 2018, 30, 8732-8738.

23 M. G. Ehrenreich, Z.-X. Zeng, S. Burger, M. R. Warren, M. W. Gaultois, J.-C. Tan and G. Kieslich, Chem. Commun., 2019, 55, 3911-3914.

24 H. Wang, H.-H. Liu, Z.-Y. Zhang, Z.-H. Liu, Z.-L. Lv, T.-W. Li, W.-W. Ju, H.-S. Li, X.-W. Cai and H. Han, npj Comput. Mater., 2019, 5, 1-9.

25 C. A. Bremner, M. Simpson and W. T. A. Harrison, J. Am. Chem. Soc., 2002, 124, 10960-10961.

26 L. J. Bourhis, O. V. Dolomanov, R. J. Gildea, J. A. K. Howard and H. Puschmann, Acta Crystallogr., Sect. A: Found. Adv., 2015, 71, 59-75.

27 O. V. Dolomanov, L. J. Bourhis, R. J. Gildea, J. A. K. Howard and H. Puschmann, J. Appl. Crystallogr., 2009, 42, 339-341.

28 G.-Q. Feng, W.-X. Zhang, L.-Y. Dong, W. Li, W.-Z. Cai, W.-J. Wei, L.-J. Ji, Z.-S. Lin and P.-X. Lu, Chem. Sci., 2019, 10, 1309-1315.

29 J. Hammersley, Fit2d User Manual, 1996.

30 M. J. Cliffe and A. L. Goodwin, J. Appl. Crystallogr., 2012, 45, 1321-1329.

31 S. J. Clark, M. D. Segall, C. J. Pickard, P. J. Hasnip, M. J. Probert, K. Refson and M. C. Payne, Z. Kristallogr., 2005, 220, 567. 
32 B. G. Pfrommer, M. Côté, S. G. Louie and M. L. Cohen, J. Comput. Phys., 1997, 131, 233-240.

33 Y. Le Page and P. Saxe, Phys. Rev. B: Condens. Matter Mater. Phys., 2002, 65, 104104.

34 H. J. Monkhorst, Phys. Rev. B: Condens. Matter Mater. Phys., 1976, 13, 5188-5192.

35 K. T. Butler, A. Walsh, A. K. Cheetham and G. Kieslich, Chem. Sci., 2016, 7, 6316-6324.

36 Z.-Y. Deng, F.-X. Wei, S.-J. Sun, G. Diesel, A. K. Cheetham and P. D. Bristowe, J. Mater. Chem. A, 2016, 4, 1202512029.
37 R. Gaelic, P. Pullman and F.-X. Coudert, J. Phys.: Condens. Matter, 2016, 28, 275201.

38 G.-Q. Feng, Y. Qin, C. Ran, L.-J. Ji, L.-Y. Dong and W. Li, APL Mater., 2018, 6, 114201.

39 L.-Y. Dong, S.-J. Sun, Z.-Y. Deng, W. Li, F.-X. Wei, Y.-J. Qi, Y.-C. Li, X.-D. Li, P.-X. Lu and U. Ramamurty, Comput. Mater. Sci., 2018, 141, 49-58.

40 Y.-G. Wang, X.-J. Lu, W.-G. Yang, T. Wen, L.-X. Yang, X.-T. Ren, L. Wang, Z.-S. Lin and Y.-S. Zhao, J. Am. Chem. Soc., 2015, 137, 11144-11149.

41 S. F. Pugh, Philos. Mag. J. Sci., 2009, 45, 823-843. 